\title{
IOL Formula Constants: Strategies for Optimization and Defining Standards for Presenting Data
}

\author{
Achim Langenbucher $^{a}$ Nóra Szentmáry $^{b, c}$ Alan Cayless $^{d}$ Michael Müller $^{\mathrm{e}}$ \\ Timo Eppig ${ }^{\mathrm{a}}$ Simon Schröder ${ }^{\mathrm{a}}$ Ekkehart Fabian $^{\mathrm{e}}$ \\ aDepartment of Experimental Ophthalmology, Saarland University, Homburg/Saar, Germany; ${ }^{b}$ Dr. Rolf M. Schwiete \\ Center for Limbal Stem Cell and Aniridia Research, Saarland University, Homburg/Saar, Germany; 'Department of \\ Ophthalmology, Semmelweis-University, Budapest, Hungary; ${ }^{d}$ School of Physical Sciences, The Open University,

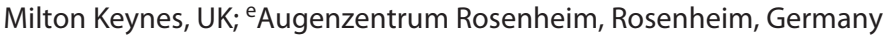

\section{Keywords \\ Lens power calculation formulae $\cdot$ Formula constant optimization · Customized constants · Non-linear optimization · Mean absolute prediction error}

\begin{abstract}
Purpose: The aim of this study is to present strategies for optimization of lens power (IOLP) formula constants and to show options how to present the results adequately. Methods: A dataset of $N=1,601$ preoperative biometric values, IOLP data and postoperative refraction data was split into a training set and a test set using a random sequence. Based on the training set, we calculated the formula constants for established lens calculation formulae with different methods. Based on the test set, we derived the formula prediction error (PE) as difference of the achieved refraction from the formula predicted refraction. Results: For formulae with 1 constant, it is possible to back-calculate the individual constant for each case using formula inversion. However, this is not possible for formulae with $>1$ constant. In these cases, more advanced concepts such as non-linear optimization strategies are necessary to derive the formula constants. During cross-validation, measures such as the mean abso-
\end{abstract}

karger@karger.com www.karger.com/ore

Karger"

BOPEN ACCESS
(C) 2021 The Author(s)

Published by S. Karger AG, Basel

This is an Open Access article licensed under the Creative Commons Attribution-NonCommercial-4.0 International License (CC BY-NC) (http://www.karger.com/Services/OpenAccessLicense), applicable to the online version of the article only. Usage and distribution for commercial purposes requires written permission. lute or the root mean squared PE or the ratio of cases within mean absolute PE (MAE) limits could be used as quality measures. Conclusions: Different constant optimization concepts yield different results. To test the performance of optimized formula constants, a cross-validation strategy is mandatory. We recommend performance curves, where the ratio of cases within absolute PE limits is plotted against the MAE.

(c) 2021 The Author(s).

Published by S. Karger AG, Basel

\section{Background}

In cataract surgery, most intraocular lens (IOL) power calculations worldwide are performed with theoreticaloptical formulae [1-3]. These formulae require biometric measurements of the patient eye before cataract surgery derived with a biometer. They also involve formula constants, which adapt the general formula to the characteristics of the specific IOL [4]. These characteristics include the optics and haptic design as well as the material properties. Depending on the philosophy of the IOL calculation formula, the constants in most of the classical formulae directly interact with the effective lens position or the lens power (IOLP) [5]. 
When an IOL company launches a new type of IOL to the market, the formula constants provided to surgeons for IOLP calculation are mostly estimated from the respective constants of similar lens types already on the market $[4,6]$, or they are derived in some pre-market studies based on a very limited number of cases. Once the lens has been on the market for a while, more clinical data are available including preoperative biometric data, the IOLP implanted, and the postoperative refractive outcome in terms of subjective sphere and cylinder or spherical equivalent. This allows the formula constants to be successively refined for better refractive outcomes in the future. This is a typical forward prediction process, where the data of previous cataract surgeries are used to predict the proper formula constant for cataract surgeries in the future.

However, there are no standards established in the literature specifying how to calculate formula constants for a specific dataset $[4,7,8]$. There is also no consensus on how many data points are required for reliable prediction of formula constants. There is also no standard for presentation of results, which would facilitate a comparison of different studies [9].

From the theory, it is clear that in all processes of forward prediction the dataset used for calculation of the formula constant should not be used for validation or verification. This implies that the dataset should be split into 2 non-overlapping sets: a training set, used for derivation of the formula constants, and a test or validation set used for verifying the performance of the formula constant in terms of a cross-validation. If validation is not performed on an independent dataset, there might be some overestimation of the performance of the formula constants [9]. There are several options for cross-validation: with only one dataset available, established techniques include "holdout," "K fold cross-validation," or "repeated random subsampling." In contrast to holdout, where the dataset is simply split into training and test sets using a binary random sequence, $\mathrm{K}$ fold cross-validation splits the data into $\mathrm{K}$ partitions and the formula constant is calculated based on K-1 partitions by excluding partition I from the calculation and then testing with the excluded partition I. This process is repeated until each partition has been excluded and used as the test partition once. With repeated random subsampling, a random partition is separated out before calculation of the formula constant and this separated dataset then used for validation of the formula constant. However, this carries the risk that some data points may never be included in the training or validation data sets [10].
Furthermore, the number of data points required for formula constant optimization is unclear. In general, with increasing number, we expect a more reliable constant if the data pool is consistent, but the number depends also on the quality of the dataset and the composition of the study population [4].

Furthermore, there is no consensus or standard by which the target parameter should be optimized, or on the appropriate norm for optimization. In simple terms, we could back-calculate an appropriate individual formula constant for each data point within a dataset, and from these individual results, we could consider, for example, the mean or median value as an optimized constant. However, taking the mean or median as the optimized formula, constant does not guarantee that the mean or median of the refraction error is zeroed. On the other hand, we could solve the formula for the predicted refraction; calculate the deviation of the achieved refraction from the formula predicted refraction considered as "prediction error" (PE) and use non-linear optimization algorithms for minimizing, for example, the mean absolute PE (MAE) or the mean root squared PE (RMSE). Different target parameters and optimization criteria yield different results for the optimized formula constant, and there is no consensus on which technique should be used [9].

There are even various options for presentation of the results, making direct comparisons difficult. In some studies, the mean and standard deviation of the PE are documented, whereas in other studies, we find results on the MAE or the MRSE. Again, in other studies, we find distribution measures of the individual formula constants such as mean, median, or standard error, or the authors document the performance curves for MAE or RMSE or the portion of eyes which are within limits of a quarter, a half, or 1 dioptre of PE.

The purpose of this study is to show, using a large dataset of a cataract population with preoperative biometric data, the IOLP of the implanted lens, and postoperative refraction data:

- How cross-validation works in a clinical setting, and how the results compare with training and validation both carried out on the entire dataset,

- What the differences are in optimizing for different target parameters or using different target criteria or norms,

- To explain the meaning of different representation formats for our results, and

- To make recommendations on which presentation format shows the results most appropriately.
Langenbucher/Szentmáry/Cayless/Müller/ Eppig/Schröder/Fabian 
Table 1. Descriptive statistics of the entire dataset with mean, SD, median, minimum and maximum, 5, and $95 \%$ quantiles ( $90 \%$ confidence intervals)

\begin{tabular}{llllllll}
\hline$N=1,452$ & AL in $\mathrm{mm}$ & ACD in $\mathrm{mm}$ & LT in $\mathrm{mm}$ & $\mathrm{R} 1$ in $\mathrm{mm}$ & R2 in mm & IOLP in D & SEQ in D \\
\hline Mean & 23.497 & 3.099 & 4.608 & 7.741 & 7.594 & 21.519 & -0.509 \\
SD & 1.082 & 0.389 & 0.422 & 0.271 & 0.271 & 2.849 & 0.859 \\
Median & 23.419 & 3.101 & 4.606 & 7.723 & 7.588 & 21.5 & -0.375 \\
Minimum & 20.635 & 2.000 & 3.345 & 6.907 & 6.434 & 8.0 & -4.0 \\
Maximum & 29.041 & 4.354 & 5.794 & 8.771 & 8.581 & 30.0 & 2.0 \\
Quantile 5\% & 22.016 & 2.461 & 3.912 & 7.236 & 7.175 & 16.5 & -2.5 \\
Quantile 95\% & 25.529 & 3.753 & 5.311 & 8.212 & 8.056 & 26.0 & 0.5 \\
\hline
\end{tabular}

AL refers to the axial length, $A C D$ to the phakic anterior chamber depth measured from the corneal front apex to the front apex of the crystalline lens, LT to the central thickness of the crystalline lens, R1 and R2 to the corneal front surface radius in the flat and steep meridian, IOLP to the refractive power of the implanted lens, and SE to the spherical equivalent of postoperative refraction. IOLP, lens power; $A L$, axial length; $L T$, lens thickness; $S D$, standard deviation.

\section{Methods}

\section{Measurement Data}

In this retrospective study, we analysed a dataset with 1,601 clinical measurements of a cataract population from Augencentrum Rosenheim, which was transferred to us. The anonymized data contained preoperative biometric data derived with the IOLMaster 700 (Carl-Zeiss-Meditec, Jena, Germany) including axial length (AL), external phakic anterior chamber depth measured from the corneal front apex to the anterior apex of the crystalline lens (ACD), lens thickness, corneal front surface radius measured in the flat (R1), and in the steep meridian (R2). In all cases, a Sensar 1 piece IOL (Johnson \& Johnson, Brunswick, NJ, USA) was implanted. Beside refractive power of the implanted lens (IOLP), the postoperative refraction (sphere and cylinder) 6-8 weeks after cataract surgery was recorded. From the total of 1,601 measurements, $N=1,452$ complete measurements with a postoperative visual acuity of 0.6 or higher were used for formula constant optimization. Eyes with missing data (mostly anterior chamber depth) were excluded. The spherical equivalent of postoperative refraction (SEQ) was calculated as sphere $+1 / 2 \cdot$ cylinder, and the mean corneal front surface radius was calculated as $R=1 / 2(\mathrm{R} 1+\mathrm{R} 2)$. The descriptive data on pre-cataract biometry, IOLP, and postoperative refraction are summarized in Table 1.

\section{Calculation Strategy}

The anonymized Excel data (.xlsx-format) were imported into MATLAB (Matlab version 2019b, The Math Works, Natick, MA, USA) for further processing. For all eyes, an AL correction according to the Cooke formula $[11,12]$ was performed. The individual formula constant was back-calculated for each case for the SRKT formula [13, 14], the Hoffer-Q formula [15, 16], the Holladay1 formula [17], and the simplified Haigis with 1 formula constant a0 and standard values for $\mathrm{a} 1=0.4$ and $\mathrm{a} 2=0.1[2,4]$. For the SRKT formula 2 different strategies were applied, one according to the concept described in the original paper $[13,14]$ (SRK2 style) and one with an inversion of the SRKT formula [4].

For all calculations, the deviation of the achieved postoperative SEQ from the formula predicted SEQ was quoted as the PE. AE in this context refers to the absolute value of $\mathrm{PE}$ and $\mathrm{SE}$ to the squared value of $\mathrm{PE}$.

For testing the formula performance and cross-validation, the $N=1,452$ data points were split randomly into a training set $(70 \%$, 1,017 cases) and a test set $(30 \%, 435$ cases) [10]. For the training set, the mean and median A constant $\left(A_{\text {mean }}\right.$ and $A_{\text {median }}$ for formula inversion and $\mathrm{A} 0_{\text {mean }}$ and $\mathrm{A} 0_{\text {median }}$ for SRK2 style calculation) for the SRKT formula, the mean and median pACD constant $\left(\mathrm{pACD}\right.$ mean and $\mathrm{pACD}_{\text {median }}$ ) for the Hoffer-Q formula, the mean and median $\mathrm{SF}$ constant $\left(\mathrm{SF}_{\text {mean }}\right.$ and $\left.\mathrm{SF}_{\text {median }}\right)$ for the Holladay1 formula, and the mean and median a 0 constant $\left(\mathrm{a} 0_{\text {mean }}\right.$ and $\mathrm{a} 00_{\text {me- }}$ dian) for the simplified Haigis formula were calculated.

In addition, a non-linear optimization algorithm (LevenbergMarquardt algorithm, $[18,19])$ was implemented and applied to derive formula constants from the training set for the SRKT $\left(A_{\mathrm{AE}}\right.$ and $\left.A_{\mathrm{SE}}\right)$, the Hoffer-Q ( $\mathrm{pACD} \mathrm{AE}_{\mathrm{AE}}$ and $\left.\mathrm{pACD} \mathrm{SE}_{\mathrm{SE}}\right)$, the Holladay1 $\left(\mathrm{SF}_{\mathrm{AE}}\right.$ and $\left.\mathrm{SF}_{\mathrm{SE}}\right)$, the simplified Haigis $\left(\mathrm{a} 0_{\mathrm{AE}}\right.$ and $\left.\mathrm{a} 0_{\mathrm{SE}}\right)$, and the Haigis formula with 3 formula constants $\left(\mathrm{a} 0 / \mathrm{a} 1 / \mathrm{a} 2_{\mathrm{AE}}\right.$ and $\left.\mathrm{a} 0 / \mathrm{a} 1 / \mathrm{a} 2_{\mathrm{SE}}\right)$ to minimize the MAE and the RMSE. For the formulae with 1 constant, the median constants $\left(A_{\text {median }}, \mathrm{pACD} \mathrm{D}_{\text {median }}, \mathrm{SF}_{\text {median }}\right.$, and $\mathrm{a} 0$ median $)$ were used for initialization for the optimization process, and for the Haigis formula, with 3 constants $\mathrm{a} 0 / \mathrm{a} 1 / \mathrm{a} 2=\mathrm{a} 0$ median $/ 0.4 / 0.1$ were used for initialization. As parameters for the Levenberg-Marquardt algorithm, we used a maximum of 100 iterations, with a damping of $1 \mathrm{e}-2$. As the exit criterion from the optimization loop, we used a tolerance for step sizes of $1 \mathrm{e}-14$ (optimization ends when last step is $<1 \mathrm{e}-14$ ), and a function tolerance of $1 \mathrm{e}-16$ (optimization ends if $\mathrm{AE}$ or SE improvement is $<1 \mathrm{e}-16)$.

In the next step, we calculated the MAE, the median absolute error and the RMSE (RMSE = square-root of SE) for each lens calculation formula and each optimized constant using the test dataset. Then for all formulae, we derived the portion of cases with $\mathrm{AE}$ $\leq 0.25 \mathrm{dpt}, \mathrm{AE} \leq 0.5 \mathrm{dpt}, \mathrm{AE} \leq 1.0 \mathrm{dpt}$, and $\mathrm{AE} \leq 2.0 \mathrm{dpt}$.

In a final step, we calculated the MAE based on the training set (used to optimize the constants) to evaluate the robustness of the optimized constants to variation of the formula constant. For the formulae with 1 constant (SRKT, Hoffer-Q, Holladay1, simplified Haigis), the formula constant optimized for the lowest RMSE of 

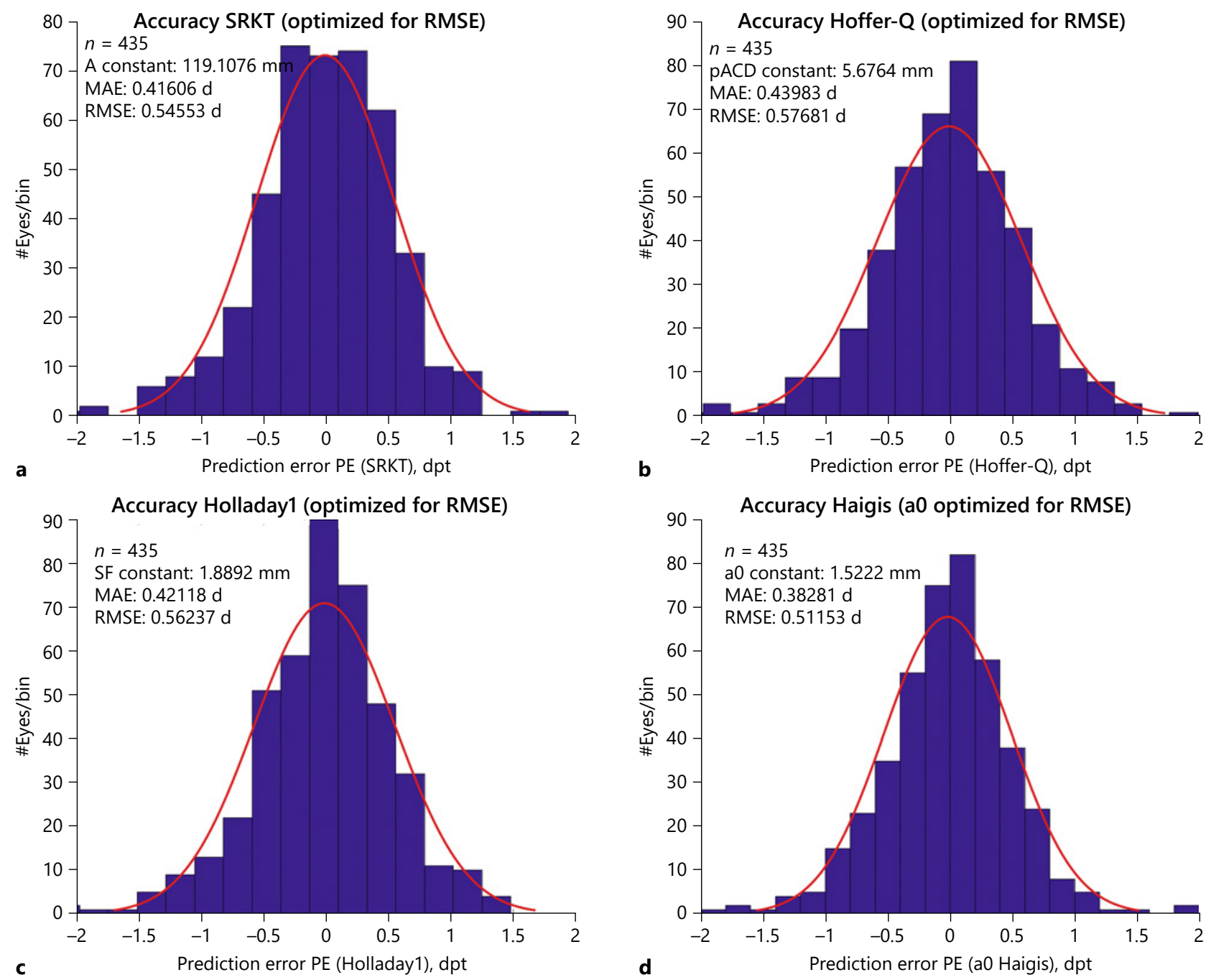

100 Accuracy Haigis (a0/a1/a2 optimized for RMSE)

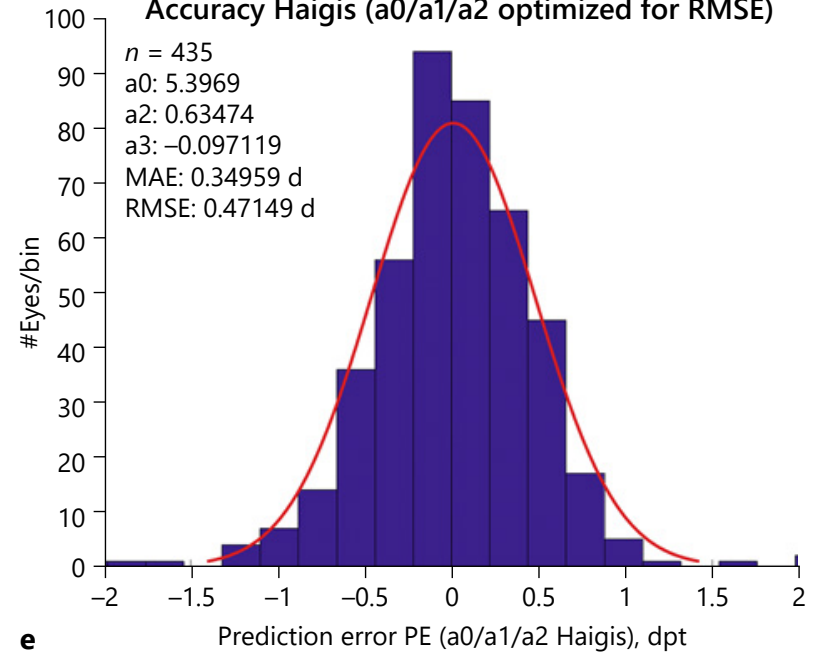

(For legend see next page.) 
refraction was varied by \pm 1 in steps of 0.01 and the portion of cases with $\mathrm{AE} \leq 0.25 \mathrm{dpt}, \mathrm{AE} \leq 0.5 \mathrm{dpt}$, and $\mathrm{AE} \leq 1.0 \mathrm{dpt}$ (in the training set) was recorded. For the Haigis formula with 3 constants (a0/a1/ a 2 ), we varied $\mathrm{a} 0$ by \pm 1 , a 1 by \pm 0.2 , and $\mathrm{a} 2$ by \pm 0.1 each in 100 equidistant steps starting from a0/a1/a $2_{\mathrm{RMSE}}$ and calculated for each constant triplet the MAE to test the robustness of the constant triplet to variations in $\mathrm{a} 0$, in $\mathrm{a} 1$, and in $\mathrm{a} 2$.

\section{Results}

The formula constants derived from the training set for all formulae under test are summarized in Table 2. For formulae with 1 formula constant (SRKT, Hoffer-Q, Holladay1, simplified Haigis with optimized a0), the mean and median of the individual formula constant are listed. For the SRKT formula, we have also provided the mean and median of the individual formula constant calculated according to the concept proposed in the original paper (SRK2 style, $\mathrm{A} 0_{\text {mean }}$ and $\mathrm{A} 0_{\text {median }}$, Lit...). In addition, for all formulae, we have listed the respective formula constants calculated from a non-linear optimization process in terms of minimizing the mean absolute and the root mean squared refraction error.

The distribution of the PE for all formulae with constant optimization for RMSE is displayed in Figure 1. Optimization was performed on the training set $(N=1,017)$, and cross-validation was done on the test set $(N=435)$.
The respective formula constants listed in Table 2. Figure 1a refers to the SRKT formula $\left(A_{\mathrm{RMSE}}\right)$, Figure $1 \mathrm{~b}$ to the

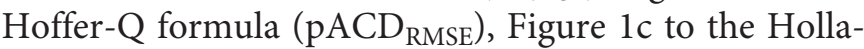
day1 formula $\left(\mathrm{SF}_{\mathrm{RMSE}}\right)$, Figure $1 \mathrm{~d}$ to the simplified Haigis formula with customized $\mathrm{a} 0$ and preset values for $\mathrm{a} 1=0.4$ and $\mathrm{a} 2=0.1\left(\mathrm{a} 0_{\text {RMSE }}\right)$, and Figure $1 \mathrm{e}$ to the Haigis formula with constant triplet ( $\left.\mathrm{a} 0 / \mathrm{a} 1 / \mathrm{a} 2_{\mathrm{RMSE}}\right)$.

The distributions of the absolute PE AE for all different constant optimization strategies and each formula are shown in Figure 2. Again, optimization was performed on the training set, and cross-validation was done on the test set. The respective formula constants listed in Table 2 . Figure $2 \mathrm{a}$ refer to the $\mathrm{AE}$ of the SRKT formula ( $\mathrm{A} 0_{\text {mean }}$, $\mathrm{A} 0_{\text {median }}, A_{\text {mean }}, A_{\text {median }}, A_{\mathrm{MAE}}$, and $\left.A_{\mathrm{RMSE}}\right)$, Figure $2 \mathrm{~b}$ to the Hoffer-Q formula ( $\mathrm{pACD}$ mean, $\mathrm{pACD}$ median, $\mathrm{pACD}$ $\mathrm{MAE}$, and $\mathrm{pACD} \mathrm{RMSE}_{\mathrm{R}}$ ), Figure $2 \mathrm{c}$ to the Holladay1 formula $\left(\mathrm{SF}_{\text {mean }}, \mathrm{SF}_{\text {median }}, \mathrm{SF}_{\mathrm{MAE}}\right.$, and $\left.\mathrm{SF}_{\mathrm{RMSE}}\right)$, Figure $2 \mathrm{~d}$ to the simplified Haigis formula with customized $\mathrm{a} 0$ and preset values for $\mathrm{a} 1=0.4$ and $\mathrm{a} 2=0.1\left(\mathrm{a} 0_{\text {mean }}, \mathrm{a} 0_{\text {median }}, \mathrm{a} 0_{\mathrm{MAE}}\right.$, and $\left.\mathrm{a} 0_{\mathrm{RMSE}}\right)$, and Figure $2 \mathrm{e}$ to the Haigis formula with constant triplet ( $\mathrm{a} 0 / \mathrm{a} 1 / \mathrm{a} 2_{\mathrm{MAE}}$ and $\left.\mathrm{a} 0 / \mathrm{a} 1 / \mathrm{a} 2_{\mathrm{RMSE}}\right)$.

The robustness of formula constants is shown in Figure 3 , in terms of the ratio of cases within PE limits (Fig. 3a-d) or MAE (Fig. 3e). For the formulae with one constant, this constant was varied in limits of \pm 1 (Fig. 3a: SRKT formula; Fig. 3b: Hoffer-Q formula; Fig. 3c: Holladay1 formula; and Fig. 3d: simplified Haigis formula

Table 2. Optimized constants for the SRKT, Hoffer-Q, Holladay1, simplified Haigis formula with optimized a0, and Haigis formula with 3 optimized constants (a0/a1/a2) based on the training set

\begin{tabular}{|c|c|c|c|c|c|}
\hline$N=1,017$ training data & SRKT formula & Hoffer-Q formula & Holladay formula & $\begin{array}{l}\text { Haigis formula a0 } \\
\text { constant }\end{array}$ & Haigis a0/a1/a2 \\
\hline Mean of individual constant & $\begin{array}{l}A_{\text {mean }}=118.981 \\
\mathrm{~A} 0_{\text {mean }}=119.307\end{array}$ & $\mathrm{pACD}$ mean $=5.636$ & $\mathrm{SF}_{\text {mean }}=1.833$ & $a 0_{\text {mean }}=1.468$ & \\
\hline Median of individual constant & $\begin{array}{l}A_{\text {median }}=118.993 \\
A 0_{\text {median }}=119.322\end{array}$ & $\mathrm{pACD}_{\text {median }}=5.611$ & $\mathrm{SF}_{\text {median }}=1.820$ & $\mathrm{a} 0_{\text {median }}=1.454$ & \\
\hline Optimized for MAE & $A_{\mathrm{MAE}}=119.016$ & $p A C D_{M A E}=5.596$ & $\mathrm{SF}_{\mathrm{MAE}}=1.819$ & $a 0_{M A E}=1.450$ & $1.720 / 0.495 / 0.076$ \\
\hline Optimized for RMSE & $A_{\text {RMSE }}=119.01$ & $\mathrm{pACD}$ RMSE $=5.606$ & $\mathrm{SF}_{\mathrm{RMSE}}=1.824$ & $\mathrm{aO}_{\mathrm{RMSE}}=1.446$ & $1.565 / 0.443 / 0.089$ \\
\hline
\end{tabular}

For the formulae with 1 constant, the optimized constant was derived from the mean and the median of the individually back-calculated constant. The individual constants for the SRKT formula were back-calculated using formula inversion $\left(A_{\text {mean }}\right.$ and $\left.A_{\text {median }}\right)$ and using the SRK2 style strategy $\left(\mathrm{A} 0_{\text {mean }}\right.$ and $\left.\mathrm{A} 0_{\text {median }}\right)$. For all formulae, the optimized constant was also derived using a non-linear optimization process for lowest MAE and RMSE. MAE, mean absolute error; RMSE, root mean squared error.

Fig. 1. PE as the deviation of achieved spherical equivalent - formula predicted refraction. Formula constants have been optimized on the training set $(N=1,017)$ and are cross-validated on the test set $(N=435)$. In this example, $\mathrm{PE}$ is shown for a formula constant optimization for minimizing the RMSE. Refers to the SRKT (a), to the Hoffer-Q (b), to the Holladay1 (c), to the simplified Haigis with optimized $\mathrm{a} 0$ and preset values for $\mathrm{a} 1=0.4$ and $\mathrm{a} 2=0.1(\mathbf{d})$, and to the Haigis formula with triplet constant (a0/a1/a2) optimization (e). PE, prediction error. 

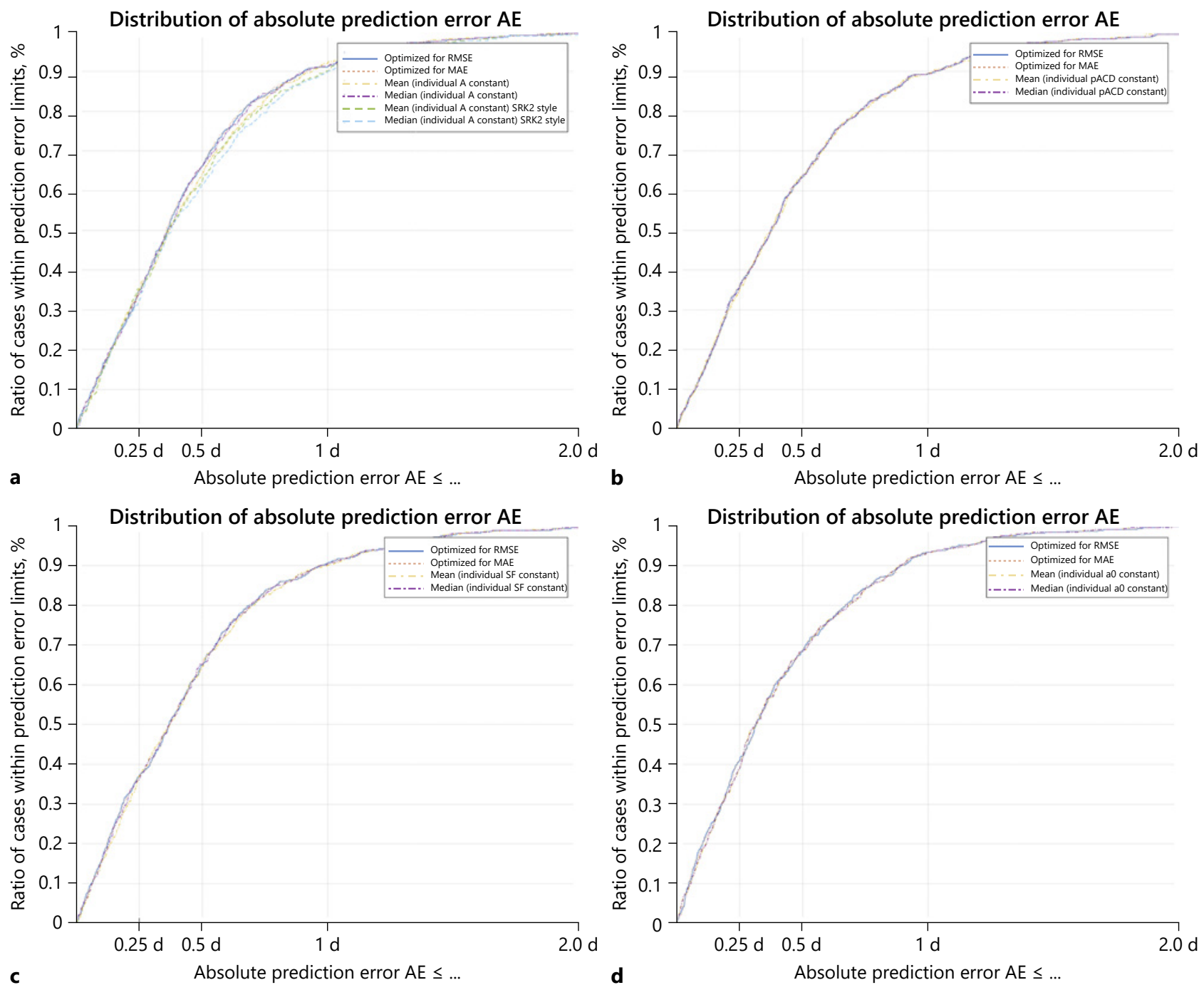

c

Absolute prediction error $\mathrm{AE} \leq \ldots$

d

Absolute prediction error $\mathrm{AE} \leq$...

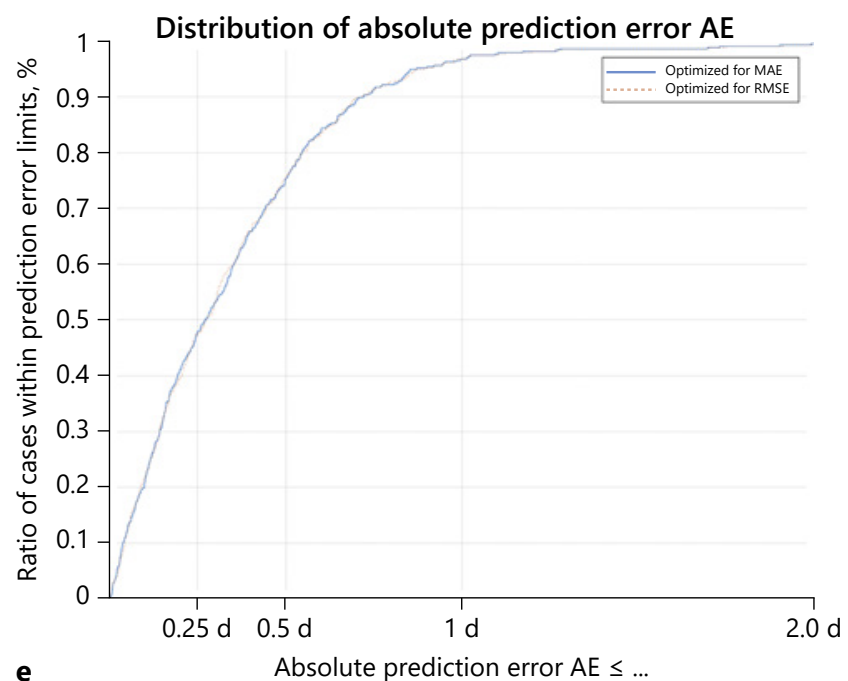

2

(For legend see next page.) 
with optimized $\mathrm{a} 0$ and $\mathrm{a} 1=0.4$ and $\mathrm{a} 1=0.1)$. For the Haigis formula with 3 formula constants (a0/a1/a2), a0 was varied in limits of \pm 1 , a 1 in limits of \pm 0.2 , and a 2 in limits of \pm 0.1 from the constant triplet optimized for RMSE (Fig. 3e). MAE data coded in colour are clipped to $\leq 2.0$ $\mathrm{dpt}$ for display. All constant triplets (a0/a1/a2) located on the blue hyperplane yield good results in terms of a low MAE.

In Figure 4, the ratio of cases within limits of absolute $\mathrm{PE} \mathrm{AE} \leq 0.25 \mathrm{dpt}, \leq 0.5 \mathrm{dpt}$, and $\leq 1.0 \mathrm{dpt}$ is displayed. Formula constants were optimized on the training set $(N=$ 1,017) for minimum RMSE and cross-validated on the test set $(N=435)$.

A comparison of all formulae under test is shown in Figure 5. The formula performance in terms of the absolute PE MAE is displayed for the SRKT, the Hoffer-Q, the Holladay1, the simplified Haigis with optimized $\mathrm{a} 0$ and standard values for a1 $=0.4$ and $\mathrm{a} 2=0.1$, and for the Haigis formula with constant triplet optimization a0/a1/a2. Optimization for minimum RMSE was performed on the training set and cross-validated on the test set.

\section{Discussion}

In the last 20 years, patient expectations for an excellent visual performance after cataract surgery have increased greatly, as cataract surgery becomes more and more standardized and complications very rare. However, there is still controversial discussion over which formula should be used for the general cases [1-3] or special situations such as long or short eyes or uncommon anterior segment geometries [7, 20,21]. General rules for selecting the "best formula" are difficult to define. There are currently many different competing calculation concepts and most of the formula authors are self-opinionated that their own philosophy of calculating the power of an IOL implant outperforms other concepts.

The key to success in formula-based IOLP calculation is the use of appropriate formula constants [2, 7, 22-25]. These constants adapt a generic formula, which is a more general formulation of a mathematical concept, to a specific IOL type, special surgery conditions, patient's eth-

Fig. 2. Performance of formula outcome in terms of MAE. Formula constants have been optimized on the training set $(N=1,017)$ and cross-validated on the test set $(N=435)$. Refers to the AE of the SRKT formula ( $\mathrm{A} 0_{\text {mean }}, \mathrm{A} 0_{\text {median }}, A_{\text {mean }}, A_{\text {median }}, A_{\mathrm{MAE}}$, and $A_{\mathrm{RMSE}}$ ) (a), to the Hoffer-Q formula ( $\mathrm{pACD}$ mean $\mathrm{pACD}$ median, $\mathrm{pACD}$ MAE, and $\mathrm{pACD} \mathrm{RMSE}_{\mathrm{R}}$ (b), to the Holladayl formula ( $\mathrm{SF}_{\text {mean }}$,

Strategies for Formula Constant

Optimization nicities, or measurement equipment. The formula constants provided by the manufacturer can be used as a good estimate or starting point for further optimization. From the basic idea, a constant optimization can be performed post hoc if results of a sufficient number of representative clinical data with a lens type have already been collected [24]. The result of the optimization process is then applied to subsequent cataract procedures in terms of a forward prediction.

Such a constant optimization process requires all biometric data which feed into the formula for IOLP calculation. In addition to the biometric data, the power of the lens and the postoperative refraction in terms of sphere and cylinder or spherical equivalent are required. For formulae with one constant, there is a straightforward option for calculating the formula constant. The formula can be re-organized and solved for the constant, and for each clinical case, we could back-calculate which formula constant is required for the biometric data together with the power of the implanted lens to yield the refraction actually achieved after cataract surgery. For each clinical case, we obtain an individual constant, and the mean or median of all individual constants could be quoted as an optimized constant for a large dataset. However, this strategy does not optimize the dataset for the refractive outcome, but rather for any of the measures in the distribution function of the individual constants.

For formulae with $>1$ constant, this simple concept of back-calculating the individual constant for each clinical case fails. For example, for the Haigis formula with 3 constants, the effective lens position $d$ can be back-calculated, and in most constant optimization strategies, a multivariate linear regression is used to calculate the constant triplet $\mathrm{a} 0 / \mathrm{a} 1 / \mathrm{a} 2$ as intercept $(\mathrm{a} 0)$ and 2 weighting factors $(\mathrm{a} 1$ and a2) from the linear regression $(d=\mathrm{a} 0+\mathrm{a} 1 \cdot \mathrm{ACD}+$ a2.AL) $[7,9,24]$. Most of the modern IOLP calculation formulae are not published, but the classical formulae such as SRKT, Hoffer-Q, Holladay1, or Haigis formula were published $>20$ years ago. Only one of these formulae (SRKT) gives some hint in the original paper as to how the lens constant should be optimized, which was considered in this paper as "SRK2 style" optimization (and the optimized constants $\mathrm{A} 0_{\text {mean }}$ and $\mathrm{A} 0_{\text {median }}$ ) in addition to

$\mathrm{SF}_{\text {median }}, \mathrm{SF}_{\mathrm{MAE}}$, and $\left.\mathrm{SF}_{\mathrm{RMSE}}\right)(\mathbf{c})$, to the simplified Haigis formula with customized $\mathrm{a} 0$ and preset values for $\mathrm{a} 1=0.4$ and $\mathrm{a} 2=0.1$ $\left(\mathrm{a} 0_{\text {mean }}, \mathrm{a}_{\text {median }}, \mathrm{a} 0_{\mathrm{MAE}}\right.$, and $\left.\mathrm{a} 0_{\mathrm{RMSE}}\right)(\mathbf{d})$, and to the Haigis formula with constant triplet (a0/a1/a $2_{\mathrm{MAE}}$ and a0/a1/a2 $2_{\mathrm{RMSE}}$ ) (e). PE, prediction error; RMSE, root mean squared error. 

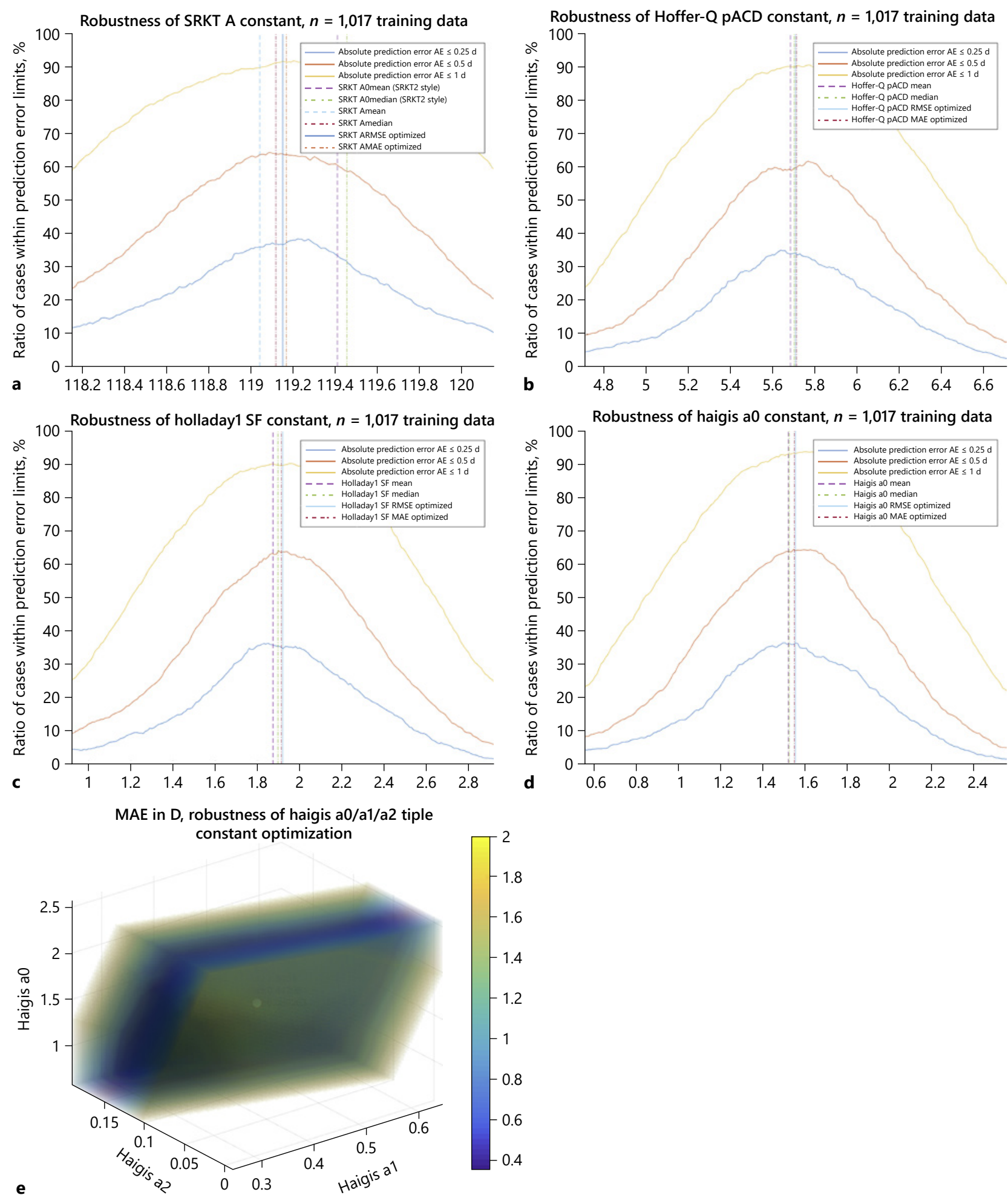
the classical formula inversion (optimized constants $A_{\text {me- }}$ an and $\left.A_{\text {median }}\right)$. There are no general rules for formula constant optimization. With steadily increasing computing capacity and speed, we are no longer bound to straightforward calculation concepts with direct back-calculation of the formula constant. Instead, non-linear optimization algorithms have been developed with a very high performance which could optimize any target parameter with any optimization criterion. This means, that instead of extracting any measure from the distribution of all individual constants, we could, for example, optimize for the mean, the mean absolute, the median, or the RMSE in terms of deviation of the achieved refraction after cataract surgery from the formula predicted refraction. In other words, we replace any measure from the statistical distribution of individual constants by a measure which has high relevance for the patient and her/his refractive outcome. In the present paper, we have implemented the Levenberg-Marquardt algorithm $[18,19]$ as a non-linear

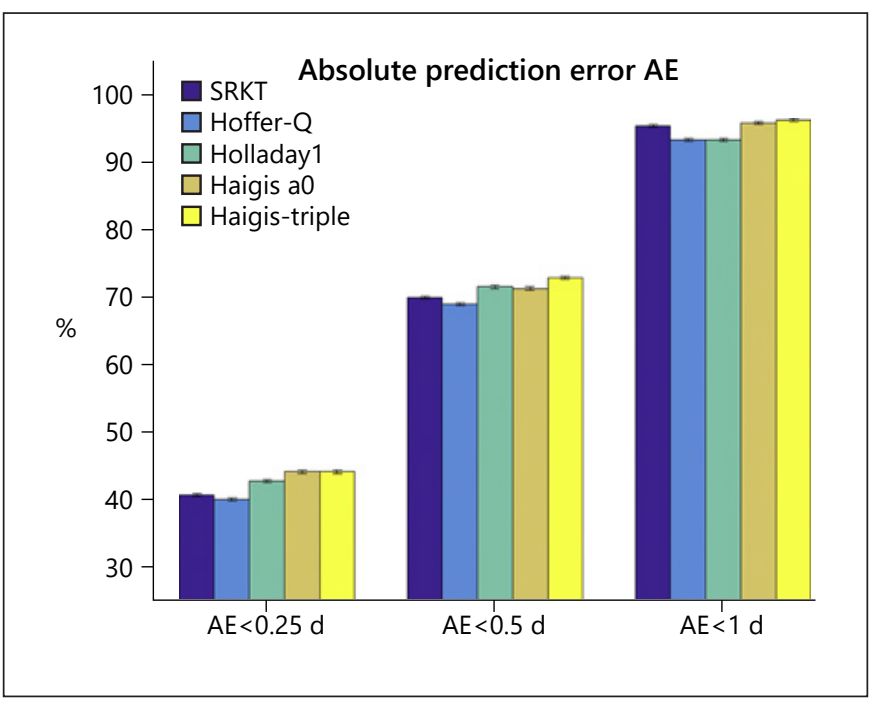

Fig. 4. Ratio of cases within limits of absolute $\mathrm{PE} \mathrm{AE} \leq 0.25 \mathrm{dpt}$, $\leq 0.5 \mathrm{dpt}$, and $\leq 1.0 \mathrm{dpt}$. Formula constants were optimized on the training set $(N=1,017)$ for minimum RMSE and cross-validated on the test set $(N=435)$. PE, prediction error; RMSE, root mean squared error.

Fig. 3. Robustness of formula constants in terms of ratio of cases within PE limits (a-d) or MAE (e). Formula constants have been optimized on the training set $(N=1,017)$ and evaluated on the training set $(N=1,017)$. For the formulae with one constant, this constant was varied in limits of \pm 1 (SRKT formula, a; Hoffer-Q formula, b; Holladay1 formula, c; simplified Haigis formula with optimized $\mathrm{a} 0$ and $\mathrm{a} 1=0.4$ and $\mathrm{a} 1=0.1, \mathbf{d})$. For the Haigis formula gradient descent method to search for an optimized constant which yields the lowest mean absolute or RMSE in addition to the straightforward calculation of the constants using formula inversion.

In reality, in most of the datasets, the difference between all the optimization strategies is expected to be low. We found only slight differences between the constants derived with formula inversion and the constants based on non-linear optimizations. However, in the case of datasets with outliers or skewed distributions of parameters instead of a "clean" dataset where data are acquired under strict study conditions (as was the case here with our data set), the difference between both strategies might be higher.

In most of the papers concerning formula constant optimization, the dataset is not split into training and test or validation data $[4,7,9,23,24]$. That means that the dataset is first used to derive the optimized formula constants, and later, the same dataset is used to test for the perfor-

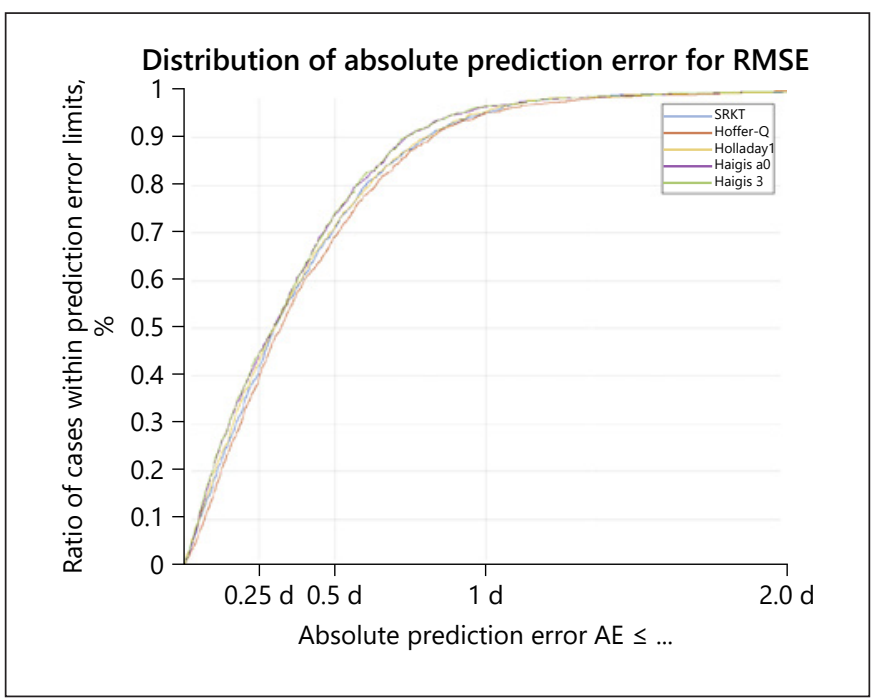

Fig. 5. Comparison of the performance of all formulae under test in terms of MAE. Formula constants were optimized on the training set $(N=1,017)$ for minimum RMSE and cross-validated on the test set $(N=435)$. PE, prediction error; MAE, mean absolute prediction error; RMSE, root mean squared error.

with 3 formula constants $(\mathrm{a} 0 / \mathrm{a} 1 / \mathrm{a} 2)$ variation of $\mathrm{a} 0$ was in limits of \pm 1 , variation of a 1 in limits of \pm 0.2 , and variation in a 2 in limits of \pm 0.1 from the constant triplet optimized for RMSE (e). Colourcoded MAE data were clipped to $\leq 2.0 \mathrm{dpt}$ for display. All constant triplets (a0/a1/a2) located on the blue hyperplane yield good results in terms of a low MAE. PE, prediction error; RMSE, root mean squared error. 
Table 3. Mean absolute, median absolute, and root mean squared refraction error of the test set for the different types of optimizations and the different formulae

\begin{tabular}{|c|c|c|c|}
\hline$N=435$ test data & $\begin{array}{l}\text { Mean absolute } \\
\text { refraction error MAE in } \\
d p t\end{array}$ & $\begin{array}{l}\text { Median absolute } \\
\text { refraction error MEDAE in } \\
\text { dpt }\end{array}$ & $\begin{array}{l}\text { Root mean squared } \\
\text { refraction error RMSE in } \\
\text { dpt }\end{array}$ \\
\hline \multicolumn{4}{|l|}{ SRKT formula } \\
\hline$A_{\text {mean }}$ & 0.391 & 0.322 & 0.517 \\
\hline$A_{\text {median }}$ & 0.390 & 0.314 & 0.516 \\
\hline $\mathrm{A}_{\text {mean }}$ & 0.428 & 0.339 & 0.567 \\
\hline $\mathrm{A}_{\text {median }}$ & 0.433 & 0.341 & 0.572 \\
\hline$A_{\mathrm{MAE}}$ & 0.388 & 0.312 & 0.515 \\
\hline$A_{\text {RMSE }}$ & 0.388 & 0.313 & 0.515 \\
\hline \multicolumn{4}{|l|}{ Hoffer-Q formula } \\
\hline $\mathrm{pACD}$ mean & 0.417 & 0.335 & 0.548 \\
\hline $\mathrm{pACD}$ median & 0.416 & 0.326 & 0.547 \\
\hline $\mathrm{pACD}$ MAE & 0.417 & 0.326 & 0.546 \\
\hline $\mathrm{pACD}$ RMSE & 0.416 & 0.326 & 0.546 \\
\hline \multicolumn{4}{|l|}{ Holladay 1 formula } \\
\hline $\mathrm{SF}_{\text {mean }}$ & 0.390 & 0.300 & 0.528 \\
\hline $\mathrm{SF}_{\text {median }}$ & 0.390 & 0.297 & 0.528 \\
\hline $\mathrm{SF}_{\mathrm{MAE}}$ & 0.390 & 0.299 & 0.528 \\
\hline $\mathrm{SF}_{\mathrm{RMSE}}$ & 0.390 & 0.299 & 0.528 \\
\hline \multicolumn{4}{|c|}{ Simplified Haigis formula with a0 } \\
\hline $\mathrm{a} 0_{\text {mean }}$ & 0.369 & 0.283 & 0.492 \\
\hline $\mathrm{aO}_{\text {median }}$ & 0.368 & 0.282 & 0.491 \\
\hline $\mathrm{a}_{\mathrm{MAE}}$ & 0.368 & 0.283 & 0.491 \\
\hline $\mathrm{aO}_{\text {RMSE }}$ & 0.368 & 0.282 & 0.491 \\
\hline \multicolumn{4}{|c|}{ Haigis formula with a0/a1/a2 } \\
\hline $\mathrm{a} 0 / \mathrm{a} 1 / \mathrm{a} 2_{\mathrm{MAE}}$ & 0.366 & 0.288 & 0.488 \\
\hline $\mathrm{a} 0 / \mathrm{a} 1 / \mathrm{a} 2_{\mathrm{RMSE}}$ & 0.366 & 0.297 & 0.495 \\
\hline
\end{tabular}

()$_{\text {mean }}$ and () median refer to the mean and median of the individual back-calculated constant, and () MAE and () RMSE $_{\text {m }}$ to the formula constant optimization for mean absolute and root mean squared refraction error. For the SRKT formula, there are 2 types of back-calculation for the individual formula constant: direct formula inversion $\left(A_{\text {mean }}\right.$ and $\left.A_{\text {median }}\right)$ and the SRK2 like style $\left(\mathrm{AO}_{\text {mean }}\right.$ and $\left.\mathrm{A} 0_{\text {median }}\right)$. RMSE, root mean squared error; MEDAE, median absolute error.

mance of the formula with the optimized constants. From all concepts in artificial intelligence or machine learning, we know that a strict separation of the dataset into training and test set is mandatory in terms of a cross-validation [10]. The entire dataset is split into training data for constant optimization and test set for validation. In this paper, we used a randomly selected $70 \%$ subset of our large dataset for training and the remaining $30 \%$ for validation. Only when testing the robustness of our constants [25] were the training data also used for testing the performance for variation of the constant; this was done to ensure that the robustness graphs have a direct clinical impact.

In this paper, we have attempted to describe different concepts of constant optimization based on a large study population of cataract patients treated with 1 intraocular lens type. All the data were collected at 1 clinical centre, and the clinical settings appear very homogeneous for this dataset as shown in Table 1. In Table 2, we have listed all optimized constants derived with different optimization strategies. For all formulae with 1 formula constant (SRKT, Hoffer-Q, Holladay1, and simplified Haigis formula), we back-calculated the individual constant by formula inversion and considered the mean and median value as the optimized constant. In addition, for the SRKT formula, we implemented the optimization strategy provided in the original paper of Sanders, Retzlaff, and Kraff $[13,14]$. For all formulae, we used a non-linear optimization method to derive the formula constants yielding the lowest MAE or the lowest root mean squared PE for the 
training set $[18,19]$. Both non-linear optimization methods are more related to the needs of the surgeon and the patients, as they both intend to result in a refraction that is closest to the formula predicted refraction. As expected, the differences between the different optimization strategies in our homogeneous dataset are generally low, and only the results of the SRK2 style constants $\mathrm{A} 0_{\text {mean }}$ and $\mathrm{A} 00_{\text {median }}$ differ significantly from the respective results of the formula inversion $A_{\text {mean }}$ and $A_{\text {median }}$ or the results of the non-linear optimization $A_{\mathrm{MAE}}$ and $A_{\mathrm{RMSE}}$.

The results for the PE using the constants optimized for the lowest RMSE are shown in the histograms of Figure 1 for all formulae. The mean and median of the PE are not necessarily zero as we did not optimize for a mean PE, but for the mean absolute or root mean squared PE. In the graphs, we have included the best-fit normal distribution and the mean absolute and RMSE. The respective values for the MAE, the median absolute error, together with the RMSE for all optimization strategies for all formulae are listed in Table 3. The MAE ranged between 0.366 and $0.433 \mathrm{dpt}$, the median absolute PE between 0.288 and $0.341 \mathrm{dpt}$, and the RMSE between 0.488 and $0.572 \mathrm{dpt}$.

To our knowledge, the robustness to variation of formula constants has not been investigated so far. For this analysis, we used the training set (previously used for constant optimization) and varied the constants starting from the optimized constant in terms of a minimum RMSE. The respective graphs for the formulae using 1 constant are shown in Figure $3 \mathrm{a}-\mathrm{d}$. The 3 curves refer to the ratio of cases within absolute $\mathrm{PE}$ limits of $\mathrm{AE} \leq 0.25$, $\leq 0.5$, and $\leq 1.0 \mathrm{dpt}$. The vertical lines indicate the various optimized constants. From the graphs, we see that the tolerance in the SRKT A constant is larger than the Hoffer-Q pACD, the Holladay 1 SF, or the a 0 of the simplified Haigis formula. This means that a certain inaccuracy of the formula constant affects the formula performance less in the SRKT formula than in other formulae. The visualization for the Haigis formula with 3 constants a $0 / a 1 /$ a2 is more complex, as the entire constant triplet affects the performance of the formula. What we see from Figure $3 \mathrm{e}$ is that there is a hyperplane in the plot where we obtain a low MAE (hyperplane in blue), and to both sides of the hyperplane, the performance is somehow degraded. That means that there is no unique solution for the constant triplet: all constant triplets located on the blue hyperplane yield acceptable results for the MAE, even if the $\mathrm{a} 0$ values and the a 1 values or the a 2 values differ significantly.

In most papers dealing with lens calculation formulae or constant optimization, the main outcome is the ratio of cases within limits of the absolute PE AE [25]. Typical limits are $\leq 0.25, \leq 0.5$, or $\leq 1.0 \mathrm{dpt}$ as shown for the crossvalidated data in Figure 4. However, with these data, we interpret only a small selection of the information from the performance curves displayed in Figure 2a-e for all formulae and optimization strategies, or for the overview with a comparison of formulae with constants optimized for the RMSE. Such performance plots are much more meaningful and provide the ratio of cases within any limit of AE. The closer the curve is to 1 the better the performance. The interpretation of such performance curves is similar to a receiver operating characteristic curve. For example, with our results shown in Figure 5, the Haigis formula with an optimized constant triplet a0/a1/a2 performs better than the Hoffer-Q formula in our test set in terms of absolute PE AE. We are nevertheless aware that the differences between formula performances in the present study are small.

Splitting the dataset into training and test sets, which is necessary for an unbiased evaluation of the formula constants in terms of cross-validation, involves some risks: first, we have to decide which portion of the dataset should be used for constant optimization. Typical values are $70 \%$ for training and 30\% for validation. In smaller datasets, we have to ensure that the number of data points used for constant optimization is sufficient to yield reliable constants, especially with formulae with $>1$ constant where the requirements on the number of data points are much higher than formulae with 1 constant. Further, data splitting is always performed with a random sequence, and depending on the selection of the training and test sets the results of cross-validation could somehow diverge. This means that repeating the entire formula constant optimization process and cross-validation using another random sequence for data splitting could produce different results. In a large dataset as in our study, the differences in the cross-validated data are not expected to depend too much on the random sequence for data splitting, but in smaller datasets, this could be a problem. However, data splitting and crossvalidation exactly reflect the situation in clinical life, where we optimize constants on an existing dataset and use the constant for predicting the IOLP in a new cataract case. For our dataset, we tested the performance of formula constants optimized in the training set for RMSE if applied on the test set, the training set, and the entire dataset. The performance in terms of ratio of cases within AE limits of $\leq 0.25, \leq 0.5$, and $\leq 1.0 \mathrm{dpt}$ is listed in Table 4 . What we see is that in most cases the optimized constants perform better in terms of a higher ratio of cases within 
Table 4. Ratio of cases within absolute PE AE limits of $\leq 0.25 \mathrm{dpt}, \leq 0.5 \mathrm{dpt}$, and $\leq 1.0 \mathrm{dpt}$ for the test set, the training set, and the entire dataset

\begin{tabular}{|c|c|c|c|}
\hline Ratio of cases within AE limits in \% & $\mathrm{AE} \leq 0.25 \mathrm{dpt}$ & $\mathrm{AE} \leq 0.5 \mathrm{dpt}$ & $\mathrm{AE} \leq 1.0 \mathrm{dpt}$ \\
\hline \multicolumn{4}{|l|}{ SRKT formula } \\
\hline Test set, $N=435$ & 40.69 & 69.89 & 95.40 \\
\hline Training set, $N=1,017$ & 41.69 & 71.49 & 95.28 \\
\hline All, $N=1,452$ & 41.39 & 71.01 & 95.32 \\
\hline \multicolumn{4}{|l|}{ Hoffer-Q formula } \\
\hline Test set, $N=435$ & 40.00 & 68.97 & 93.33 \\
\hline Training set, $N=1,017$ & 39.43 & 69.42 & 95.97 \\
\hline All, $N=1,452$ & 39.60 & 69.28 & 95.18 \\
\hline \multicolumn{4}{|l|}{ Holladay 1 formula } \\
\hline Test set, $N=435$ & 42.75 & 71.49 & 93.33 \\
\hline Training set, $N=1,017$ & 43.26 & 70.70 & 96.56 \\
\hline All, $N=1,452$ & 43.11 & 70.94 & 95.59 \\
\hline \multicolumn{4}{|l|}{ Simplified Haigis formula with a0 } \\
\hline Test set, $N=435$ & 44.14 & 71.26 & 95.86 \\
\hline Training set, $N=1,017$ & 44.84 & 74.83 & 96.85 \\
\hline All, $N=1,452$ & 44.63 & 73.76 & 96.56 \\
\hline \multicolumn{4}{|l|}{ Haigis formula with a0/a1/a2 } \\
\hline Test set, $N=435$ & 44.14 & 72.87 & 96.32 \\
\hline Training set, $N=1,017$ & 45.03 & 74.53 & 96.76 \\
\hline All, $N=1,452$ & 44.63 & 73.76 & 96.56 \\
\hline
\end{tabular}

Constants were optimized for minimum RMSE on the training set $(N=435)$. In most cases, the formula performance tested on the training set outperforms the respective performance on the test set. $\mathrm{PE}$, prediction error; RMSE, root mean squared error.

limits on the training set compared to the test set. This result seems obvious. The higher the coherence between the training and test set the closer the results for the training and test set. From a mathematical standpoint, the more individual a IOLP calculation scheme is in terms of degrees of freedom, the better the formula would be expected to reproduce the training set with the optimized constants. However, cross-validation with a mutually exclusive test set might lead to an overfitting with the consequence that the performance might be degraded significantly. As we deal with a large dataset in this study and with formulae with 1 or 3 constants, the effect of overfitting is low.

In conclusion, this study shows that:

1. For IOL calculation formulae which are disclosed and public domain, there are different strategies of formula constant optimizations. For formulae with 1 constant, we could back-calculate an individual formula constant for each case and derive the optimized constant from the mean or median of the individual constants. For formulae with $>1$ constant, the constant optimization is more difficult as we cannot back-calculate the appropriate constant for each case.
2. In addition to classical constant optimization strategies, we could always implement a non-linear optimization strategy, where any target parameter is optimized for any target criterion. An optimization for the minimal PE seems to be closest to the needs of the surgeon and patients as the predictability of the refractive outcome is the most important quality criterion in modern cataract surgery. Typical target criteria in most cases are the minimization of the RMSE or the MAE.

3. Evaluating the results of formula constant optimization always requires cross-validation. For such a crossvalidation, the dataset has to be split into a training set used for calculating the constants, and a test set used for validation of the constants. Without cross-validation, in most cases, the performance of the constants would be overestimated.

4. There are different options for presenting the results of formula constant optimization. We feel that performance curves which show the ratio of cases within limits of the absolute PE are the most appropriate tools and yield more information than the MAE or the ratio of cases within selected absolute PE limits.
Langenbucher/Szentmáry/Cayless/Müller/ Eppig/Schröder/Fabian 


\section{Statement of Ethics}

This retrospective review of patient data did not require ethical approval in accordance with local guidelines from Ethics Committee of the Bavarian State Chamber of Physicians. Written informed consent from participants was not required in accordance with local guidelines.

\section{Conflict of Interest Statement}

The authors have no conflicts of interest to declare.

\section{Funding Sources}

Not applicable.

\section{Author Contributions}

Achim Langenbucher: planning of the study, calculations and programming, and scientific writing. Nóra Szentmáry: planning of the study, clinical assistance, and critical revision. Timo Eppig: Assistance in programming and data interpretation. Simon Schröder: Assistance in programming and data interpretation. Alan Cayless: Data interpretation, scientific writing, and critical revision. Ekkehard Fabian: Data collection and clinical setting.

\section{Data Statement}

The anonymized raw data that were analyzed in the study could be provided on request.

\section{References}

1 Melles RB, Kane JX, Olsen T, Chang WJ. Update on intraocular lens calculation formulas. Ophthalmology. 2019;126(9):1334-5. Epub 2019 Apr 11. PMID: 30980854.

2 Shammas HJ. Intraocular lens power calculations: Slack Inc; 2004.

3 Olsen T. Calculation of intraocular lens power: a review. Acta Ophthalmol Scand. 2007; 85(5):472-85. Epub 2007 Apr.

4 Schröder S, Leydolt C, Menapace R, Eppig T, Langenbucher A. Determination of personalized IOL-constants for the haigis formula under consideration of measurement precision. PLoS One. 2016;11(7):e0158988.

5 Olsen T. J Prediction of the effective postoperative (intraocular lens) anterior chamber depth. J Cataract Refract Surg. 2006;32(3): $419-24$.

6 Langenbucher A, Eppig T, Viestenz A, Seitz B, Müllner G, Schönherr U. Individualisierung der IOL-Konstanten für 2 hydrophobe Intraokularlinsen. SRK II, SRK/T, Hoffer Q, Holladay 1 und Haigis-Formel [Individualization of IOL constants for two hydrophobic intraocular lenses. SRK II, SRK/T, Hoffer-Q, Holladay 1 and Haigis formula]. Ophthalmologe. 2012;109(5):468-73. PMID: 22581048.

7 Petermeier K, Gekeler F, Messias A, Spitzer MS, Haigis W, Szurman P. Intraocular lens power calculation and optimized constants for highly myopic eyes. J Cataract Refract Surg. 2009;35(9):1575-81.

8 Zhang JQ, Zou XY, Zheng DY, Chen WR, Sun A, Luo LX. Effect of lens constants optimization on the accuracy of intraocular lens power calculation formulas for highly myopic eyes. Int J Ophthalmol. 2019;12(6):943-8.
9 Schröder S, Wagenpfeil S, Leydolt C, Menapace R, Langenbucher A. [Interpretation of the intraocular lens constants for the Haigis formula]. Klin Monbl Augenheilkd. 2017; 234(8):975-8

10 Langenbucher A, Szentmáry N, Wendelstein J, Hoffmann P. Artificial intelligence, machine learning and calculation of intraocular lens power. Klin Monbl Augenheilkd. 2020 Dec;237(12):1430-7. English, German. Epub ahead of print. PMID: 33231277.

11 Cooke DL, Cooke TL. Approximating sumof-segments axial length from a traditional optical low-coherence reflectometry measurement. J Cataract Refract Surg. 2019 Mar; 45(3):351-4.

12 Cooke DL, Cooke TL. A comparison of two methods to calculate axial length. J Cataract Refract Surg. 2019;45(3):284-92.

13 Retzlaff JA, Sanders DR, Kraff MC. Development of the SRK/T intraocular lens implant power calculation formula. J Cataract Refract Surg. 1990;16(3):333-40. PMID: 2355321.

14 Sanders DR, Retzlaff JA, Kraff MC, Gimbel HV, Raanan MG. Comparison of the SRK/T formula and other theoretical and regression formulas. J Cataract Refract Surg. 1990;16(3): 341-6. PMID: 2355322.

15 Hoffer KJ. Steps for IOL power calculation. J Am Intraocul Implant Soc. 1980;6(4):370.

16 Hoffer KJ. The Hoffer Q formula: a comparison of theoretic and regression formulas. J Cataract Refract Surg. 1993;19(6):700-12. PMID: 8271165.
17 Holladay JT, Prager TC, Chandler TY, Musgrove $\mathrm{KH}$, Lewis JW, Ruiz RS. A three-part system for refining intraocular lens power calculations. J Cataract Refract Surg. 1988;14(1): 17-24. PMID: 3339543.

18 Levenberg K. A method for the solution of certain non-linear problems in least squares. Quart Appl Math. 1944;2(2):164-8.

19 Marquardt DW. An algorithm for leastsquares estimation of nonlinear parameters. Soc Ind Appl Math. 1963;11(2):431-41.

20 Hoffer KJ. Intraocular lens calculation: the problem of the short eye. Ophthalmic Surg. 1981;12(4):269-72. PMID: 7254770.

21 Abulafia A, Barrett GD, Rotenberg M, Kleinmann G, Levy A, Reitblat O, et al. Intraocular lens power calculation for eyes with an axial length greater than $26.0 \mathrm{~mm}$ : comparison of formulas and methods. J Cataract Refract Surg. 2015;41(3):548-56. Epub 2015 Feb 21.

22 Olsen T, Hoffmann P. C constant: new concept for ray tracing-assisted intraocular lens power calculation. J Cataract Refract Surg. 2014;40(5):764-73.

23 Olsen T, Thim K, Corydon L. Theoretical versus SRK I and SRK II calculation of intraocular lens power. J Cataract Refract Surg. 1990; 16(2):217-25. PMID: 2329481.

24 Aristodemou P, Knox Cartwright NE, Sparrow JM, Johnston RL. Intraocular lens formula constant optimization and partial coherence interferometry biometry: refractive outcomes in 8,108 eyes after cataract surgery. J Cataract Refract Surg. 2011;37(1):50-62.

25 Cooke DL, Cooke TL. Effect of altering lens constants. J Cataract Refract Surg. 2017;43(6): 853. PMID: 28732622. 